\title{
A Marketing Decision Problem in a Periodic Review Model with Exponential Demand and Shortages
}

\author{
Nirmal Kumar Duari ${ }^{1}$ and Prof. Tripti Chakraborty ${ }^{2}$ \\ Department of Applied Mathematics University of Calcutta 92, A P C Road, Kolkata-700009, India
}

\begin{abstract}
The paper considers a marketing decision problem in a periodic review model. The demand distribution is assumed to be exponential, with the mean demand being a concave increasing function of the level of marketing effort. The optimum ordering policy and the optimum level of marketing are determined so as to minimize the total expected cost over a re-order interval. Optimum decision rules are given for some particular functional forms of the mean demand.
\end{abstract}

\section{Introductio :}

Marketing management is one of the major components of business management. It is a business discipline which focuses on the practical application of marketing techniques and the management of a firm's marketing resources and activities Marketing technique includes advertising, distribution, communication, selling, etc. and, owing to the growing competition, a rapid growth in it is observed in various business organizations.

A marketing policy is expected to induce increase in demand and sale. As such, it is likely that the inventory maintained by a business will be affected by the marketing policy adopted. Till date, few researchers have investigated inventory models with demand dependent on the marketing strategy. See, for example, Ladany and Sternlieb (1974), Lal and Staelin (1984), Jucker and Rosenblatt (1985), Shah and Jha (1991), Eliashberg and Steinberg (1993), Bhunia and Maiti (1997), Pal and Manna (2003).

Advertisement is a common marketing strategy. It is generally observed that as the frequency of advertisement $m$ increases, the demand for a product also increases, but at a decreasing rate. Pal and Manna (2003) incorporated this feature of the marketing effort in their study of the classical single period stochastic inventory model. However, they assumed $m$ to be continuous, which is not true. In this paper, we consider a dynamic risk inventory model allowing shortage, where mean demand is assumed to be an increasing concave function of the frequency of advertisement, but with a diminishing rate. Demand is assumed to have a oneparameter exponential distribution. The paper is organized as follows. The problem is formulated in section 2. In section 3, the main results are given. In section 4, different functional forms of demand in terms of marketing effort are considered, and section 5 . Gives the concluding remarks.

\section{The Problem And Its Formulation :}

Consider a dynamic risk inventory model. The policy is to place an order at each reorder point, the order quantity being sufficient to bring up the stock height to a level $S$. The model is governed by the following assumptions:

1) Reorder points are equidistant on the planning horizon.

2) Demand $(X)$ during a reorder interval is a non-negative, continuous random variable With mean demand dependent on the level of marketing effort.

3) The marketing effort is measured by the number of units of advertisements $m$.

4) $\mu_{m}$ Denotes the mean demand during a reorder period.

5) $\mu_{m}$ is an increasing integer concave function of $m$, that is, $\mu_{m}$ increases with $m$, but the rate of increase is non-increasing. Mathematically, the assumption means

$$
\mu_{m+1}-\mu_{m}>0, \quad \mu_{m+2}-2 \mu_{m+1}+\mu_{m} \leq 0 .
$$

6) The pdf and cdf of $X$ are $f\left(x ; \mu_{m}\right)$ and $F\left(x ; \mu_{m}\right)$, respectively, $x \geq 0$.

7) Supply is instantaneous on ordering.

8) The costs are

(i) $C_{1}=$ Carrying cost per unit per unit time

(ii) $C_{2}=$ Shortage cost per unit short per unit time

(iii) $r=$ cost of an advertisement 
The problem is to find $S$ and $m$ so as to minimize the total expected cost over a reorder interval.

The cost function over a re-order interval is given by

$$
\begin{aligned}
C(S, m \mid x) \quad & =c x+r m+C_{1}(S-x / 2), \text { if } \mathrm{x} \leq S \\
= & c x+r m+C_{1} S^{2} / 2 x+C_{1}(x-S)^{2} / 2 x, \text { if } \mathrm{x} \geq S
\end{aligned}
$$

Hence, the expected cost over a reorder interval comes out to be

$$
\begin{aligned}
C(S, m)=c \mu_{m}+r m+C_{1} \int_{0}^{S}(S-x / 2) & f\left(x ; \mu_{m}\right) d x+C_{1} S^{2} \int_{S}^{\infty}(1 / 2 x) f\left(x ; \mu_{m}\right) d x \\
& +C_{2} \int_{S}^{\infty}\left\{(x-S)^{2} / 2 x\right\} f\left(x ; \mu_{m}\right) d x
\end{aligned}
$$

The problem is to find values of $m$ and $S$ that minimize $C(S, m)$.

\section{Main Result :}

For given $m$, the optimal value of $S$, which minimizes (2.1), satisfies $\frac{\partial}{\partial S} C(S, m)=0$, which gives

$$
F\left(S ; \mu_{m}\right)+S \int_{S}^{\infty} \frac{1}{x} f\left(x ; \mu_{m}\right) d x=\frac{C_{2}}{C_{1}+C_{2}}
$$

As left hand side of (2.2) is an increasing function of $S$ for given $m,(2.2)$ gives a unique solution of $S$ in terms of $m$.

Now, differentiating (2.2) with respect to $\mu_{m}$, we get

$$
\frac{\partial S}{\partial \mu_{m}}=-\left[1+S f\left(S ; \mu_{m}\right)+\int_{0}^{S} \frac{1}{2 x} f\left(x ; \mu_{m}\right) d x\right]^{-1} \int_{0}^{S}\left(1+\frac{S}{2 x}\right) \frac{\partial}{\partial \mu_{m}} f\left(x ; \mu_{m}\right) d x
$$

(2.3) means that the optimal value of $S$ is a decreasing function of $\mu_{m}$ if $\frac{\partial}{\partial \mu_{m}} f\left(x ; \mu_{m}\right)>0$ for all $x \geq 0$, and is an increasing function of $\mu_{m}$ if $\frac{\partial}{\partial \mu_{m}} f\left(x ; \mu_{m}\right)<0$ for all $x \geq 0$. Then, using assumption (5), we have the following theorem:

Theorem 2.1: If $S_{m}$ be the optimum value of $S$ minimizing $C(S, m)$ for given $m$, then $S_{m}$ is a decreasing function of $m$ if $\frac{\partial}{\partial \mu_{m}} f\left(x ; \mu_{m}\right)>0$ for all $x \geq 0$, and $S_{m}$ is an increasing function of $m$ if $\frac{\partial}{\partial \mu_{m}} f\left(x ; \mu_{m}\right)<0$ for all $x \geq$ 0.

Let $S_{m}$ be the optimum value of $S$ minimizing $C(S, m)$ for given $m$. Then, $C\left(S_{m}, m\right)$ is the minimum value

\begin{tabular}{|c|c|c|c|c|c|c|}
\hline $\mathrm{C}_{1}$ & $\mathrm{C}_{2}$ & $\mathrm{~m}$ & $\alpha$ & $\mu=\mathrm{m}^{\wedge} \alpha$ & $\mathrm{s}=0.3 * \mu$ & $c(s, \mu)$ \\
\hline 5 & $=$ & 1 & 0.3 & 1 & 0.3 & 0.559106 \\
\hline 5 & ( & 2 & 0.3 & 1.231144 & 0.32 & 0.523883 \\
\hline 5 & ( & 3 & 0.3 & 1.390389 & 0.34 & 0.508899 \\
\hline 5 & ( & 4 & 0.3 & 1.515717 & 0.36 & 0.502055 \\
\hline 5 & ( & 5 & 0.3 & 1.620657 & 0.38 & 0.499874 \\
\hline$\overline{5}$ & ( & $\overline{6}$ & $\overline{0.3}$ & 1.71177 & $\overline{0.4}$ & 0.500867 \\
\hline 5 & ( & 7 & 0.3 & 1.79279 & 0.42 & 0.504249 \\
\hline 5 & ( & 8 & 0.3 & 1.866066 & 0.44 & 0.509546 \\
\hline 5 & ( & 9 & 0.3 & 1.933182 & 0.46 & 0.516445 \\
\hline 5 & ( & 10 & 0.3 & 1.995262 & 0.48 & 0.524725 \\
\hline 5 & ( & 11 & 0.3 & 2.053136 & 0.5 & 0.534219 \\
\hline 5 & ( & 12 & 0.3 & 2.107436 & 0.52 & 0.544802 \\
\hline 5 & ( & 13 & 0.3 & 2.158654 & 0.54 & 0.556372 \\
\hline
\end{tabular}
of $C(S, m)$, for given $m$. We, therefore, obtain the optimum value of $m$ by minimizing $C\left(S_{m}, m\right)$ with respect to $m$. This means that optimum $m$ is the smallest value of $m$ for which $\quad C\left(S_{m+1}, m+1\right)-C\left(S_{m}, m\right) \geq 0$.

\section{Numerical Example:}

1. Let us consider the mean demand function, depend on $\mathrm{m}$, and be $\mu=m^{\alpha}, 0<\alpha<1$. Then the minimum cost is given from the table: 
A Marketing Decision Problem in a Periodic Review model with Exponential Demand and Shortages

\begin{tabular}{lllllll}
5 & 6 & 14 & 0.3 & 2.207183 & 0.56 & 0.568846 \\
5 & 6 & 15 & 0.3 & 2.253343 & 0.58 & 0.582155 \\
\hline
\end{tabular}

Therefore the minimized cost $C(S, m)=0.499874$ at optimized $\mathrm{m}=5$, for $\mathrm{c}_{1}=5, \mathrm{c}_{2}=6$ and $\alpha=0.3, \mathrm{~s}=0.38$.

2. Let us consider the mean demand function, depend on $\mathrm{m}$, be $\mu=A-\mathrm{B}^{*}\left({ }^{\wedge} \mathrm{m}\right)$. Then the minimized cost is given from the table:

\begin{tabular}{|c|c|c|c|c|c|c|}
\hline$\underline{\mathrm{m}}$ & A & B & g & $\mu=\mathrm{A}-\mathrm{B}^{*\left({ }^{\wedge} \wedge \mathrm{m}\right)}$ & $S=0.29^{*} \underline{\mu}$ & $c(s, \mu)$ \\
\hline 1 & 1 & 1 & 0.23 & 0.77 & 0.2233 & 0.593159 \\
\hline 2 & 2 & 2 & 0.23 & 1.8942 & 0.549318 & 0.591214 \\
\hline 3 & 3 & 2 & 0.23 & 2.975666 & 0.862943 & 0.819411 \\
\hline 4 & 4 & 3 & 0.23 & 3.991605 & 1.157565 & 1.135291 \\
\hline 5 & 5 & 3.5 & 0.23 & 4.997747 & 1.449347 & 1.519608 \\
\hline 6 & 6 & 4.25 & 0.23 & 5.999371 & 1.739818 & 1.962337 \\
\hline 7 & 7 & 4.875 & 0.23 & 6.999834 & 2.029952 & 2.458924 \\
\hline 8 & 8 & 5.5625 & 0.23 & 7.999956 & 2.319987 & 3.006369 \\
\hline 9 & 9 & 6.21875 & 0.23 & 8.999989 & 2.609997 & 3.602659 \\
\hline
\end{tabular}

Therefore

The minimized cost

$C(S, m)=0.5912144$, at optimized $\mathrm{m}=2$.

\section{Different Function Form In Terms Of Marketting Effort :}

In this portion we consider different functional form of demand in terms of marketing effort. Here our aim to find out the variation of cost function with different values of one parameter, when the other parameters are remains fixed.

Firstly, consider the mean demand function as $\mu=m^{\alpha}$ Then,

For fixed $\alpha$ and $\mathrm{C}_{2}$ :

\begin{tabular}{|l|l|l|l|l|}
\hline $\mathrm{C}_{1}$ & Optimized $\mathrm{m}$ & Optimized S & $\mu=m^{\alpha}$ & Min C $(\mathrm{s}, \mathrm{m})$ \\
\hline 2 & 5 & 0.38 & 1.620657 & 0.295329 \\
\hline 5 & 5 & 0.38 & 1.620657 & 0.499874 \\
\hline 10 & 5 & 0.38 & 1.620657 & 0.670329 \\
\hline 15 & 5 & 0.38 & 1.620657 & 0.759614 \\
\hline 20 & 5 & 0.38 & 1.620657 & 0.814559 \\
\hline
\end{tabular}

From this table we see that the cost increases with $c_{1}$. i.e., cost varies directly with $c_{1}$.

For fixed $\alpha$ and $\mathrm{C}_{1}$ :

\begin{tabular}{|l|l|l|l|l|}
\hline $\mathrm{C}_{2}$ & Optimized $\mathrm{m}$ & Optimized S & $\mu=m^{\alpha}$ & Min C(s,m) \\
\hline 2 & 5 & 0.38 & 1.620657 & 0.759614 \\
\hline 3 & 5 & 0.38 & 1.620657 & 0.670329 \\
\hline 6 & 5 & 0.38 & 1.620657 & 0.499874 \\
\hline 9 & 5 & 0.38 & 1.620657 & 0.402472 \\
\hline 12 & 5 & 0.38 & 1.620657 & 0.339446 \\
\hline 15 & 5 & 0.38 & 1.620657 & 0.295329 \\
\hline
\end{tabular}

indirectly with $\mathrm{c}_{2}$.

From this table we see that the cost decreases with the increasing values of $c_{2}$. i.e., cost varies

For fixed $\mathrm{C}_{1}$ and $\mathrm{C}_{2}$ :

\begin{tabular}{|l|l|l|l|l|}
\hline$\alpha$ & Optimized $\mathrm{m}$ & Optimized $\mathrm{S}$ & $\mu=m^{\alpha}$ & Min C(s,m) \\
\hline 0.1 & 2 & 0.32 & 1.0711773 & 0.553118 \\
\hline 0.2 & 4 & 0.36 & 1.319508 & 0.52942 \\
\hline 0.3 & 5 & 0.38 & 1.620657 & 0.499874 \\
\hline 0.4 & 6 & 0.40 & 2.047673 & 0.469934 \\
\hline 0.5 & 7 & 0.42 & 2.645751 & 0.442182 \\
\hline
\end{tabular}

Thus, the cost decreases with the increasing values of $\alpha$. i.e., cost varies indirectly with $\alpha$. 
Secondly, considering the mean demand function as $\mu=A-B^{*}\left({ }^{\natural} \wedge m\right)$. Then,

For fixed ${ }^{\mathrm{n}}$ and $\mathrm{C}_{2}$ :

\begin{tabular}{|l|l|l|l|l|}
\hline $\mathrm{C}_{1}$ & Optimized $\mathrm{m}$ & Optimized $\mathrm{S}$ & $\mu=\mathrm{A}-\mathrm{B} *(\mathfrak{\eta} \wedge \mathbf{m})$ & $\operatorname{Min} \mathrm{C}(\mathrm{s}, \mathrm{m})$ \\
\hline 2 & 2 & 0.549318 & 1.8942 & 0.386668 \\
\hline 5 & 2 & 0.549318 & 1.8942 & 0.591214 \\
\hline 10 & 2 & 0.549318 & 1.8942 & 0.761668 \\
\hline 15 & 2 & 0.549318 & 1.8942 & 0.850954 \\
\hline 20 & 2 & 0.549318 & 1.8942 & 0.905899 \\
\hline
\end{tabular}

From this table we see that the cost increases with $\mathrm{c}_{1}$. i.e., cost varies directly with $\mathrm{c}_{1}$.

For fixed ${ }^{\mathrm{v}}$ and $\mathrm{C}_{1}$ :

\begin{tabular}{|l|l|l|l|l|}
\hline $\mathrm{C}_{2}$ & Optimized $\mathrm{m}$ & Optimized $\mathrm{S}$ & $\mu=\mathrm{A}-\mathbf{B}^{*}\left({ }^{\wedge} \wedge \mathbf{m}\right)$ & $\operatorname{Min} \mathrm{C}(\mathrm{s}, \mathrm{m})$ \\
\hline 2 & 2 & 0.549318 & 1.8942 & 0.850954 \\
\hline 3 & 2 & 0.549318 & 1.8942 & 0.761668 \\
\hline 6 & 2 & 0.549318 & 1.8942 & 0.591214 \\
\hline 9 & 2 & 0.549318 & 1.8942 & 0.493811 \\
\hline 12 & 2 & 0.549318 & 1.8942 & 0.430786 \\
\hline 15 & 2 & 0.549318 & 1.8942 & 0.386668 \\
\hline
\end{tabular}

indirectly with $\mathrm{c}_{2}$.

From this table we see that the cost decreases with the increasing values of $c_{2}$. i.e., cost varies

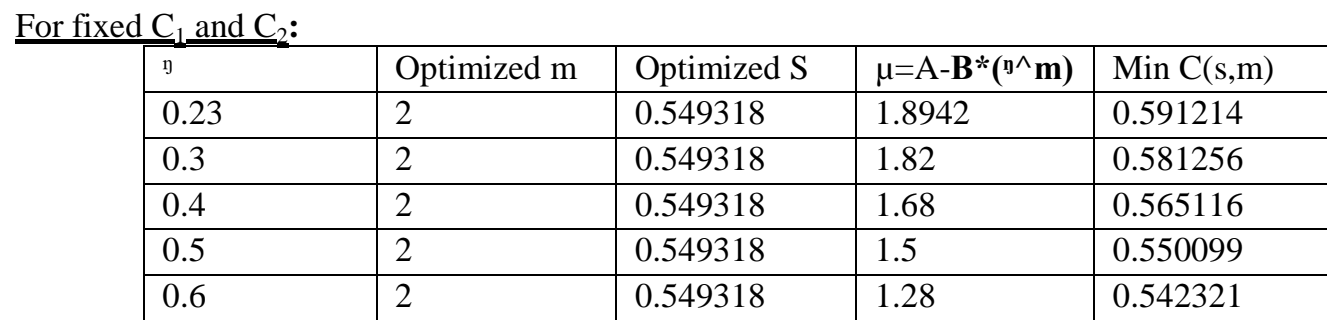

Thus, the cost decreases with the increasing values of $\eta$. i.e., cost varies indirectly with $\eta$.

\section{Concluding Remark :}

In this paper, we consider a dynamic risk inventory model allowing shortage, where mean demand is assumed to be an increasing concave function of the frequency of advertisement, but with a diminishing rate. Demand is assumed to have a one-parameter exponential distribution. A marketing policy is expected to induce increase in demand and sale. As such, it is likely that the inventory maintained by a business will be affected by the marketing policy adopted. Till date, few researchers have investigated inventory models with demand dependent on the marketing strategy. Thereby, this helps us to take decision about marketing straight or marketing policies. From this model, we can say that number of advertisement is one of the most important things and different costs have impact on total cost of the model. In future, researchers can do more work about several parts of marketing, like demand, communication, selling etc.

\section{Acknowledgement :}

The author greatly acknowledgement the referees and his guider for constructive suggestions to improve the quality of the paper.

\section{References}

[1]. Bhunia, A.K. and Maiti, M. (1997): An inventory model for decaying items with selling price, frequency of advertisement and linearly dependent demand with shortages, IAPQR Transactions, 22, 1-49.

[2]. Eliashberg, J. and Steinberg, M.S. (1993): Marketing production joint decision making, Handbook in OR and MS, Vol. 5 (Eds. J. Eliashberg and G.L. Lilien). Elsevier, Amsterdam.

[3]. Jucker, J.K. and Rosenblatt, M.J. (1985): A single period inventory model with demand uncertainty and quantity discounts: behavioural implications and a new solution procedure, Naval Research Logistic Quarterly, 32, 537-550.

[4]. Ladany, S. and Sternlieb, A (1974): The interaction of economic ordering quantities and marketing policies, AIIE Transactions, 6, $35-40$.

[5]. Lal, R. and Staelin, R. (1984): An approach for developing an optimal discount pricing policy, Management Science, 30, 15241539.

[6]. Pal and Manna (2003): A marketing decision problem in a single period stochastic inventory model, Opsearch, 40(3), 230-240.

[7]. Shah, Y.K. and Jha, P.J. (1991): A single-period stochastic inventory model under the influence of marketing policies, Journal of the Operational Research Society, 42, 173-176. 